\title{
Engineering hybrid epitaxial InAsSb/AI nanowires for stronger topological protection
}

Sestoft, Joachim E.; Kanne, Thomas; Gejl, Aske Nørskov; von Soosten, Merlin; Yodh, Jeremy S.; Sherman, Daniel; Tarasinski, Brian; Wimmer, Michael; Johnson, Erik; Deng, Mingtang

Total number of authors:

14

Published in:

Physical Review Materials

Link to article, DOI:

10.1103/PhysRevMaterials.2.044202

Publication date:

2018

Document Version

Publisher's PDF, also known as Version of record

Link back to DTU Orbit

Citation (APA):

Sestoft, J. E., Kanne, T., Gejl, A. N., von Soosten, M., Yodh, J. S., Sherman, D., Tarasinski, B., Wimmer, M., Johnson, E., Deng, M., Nygard, J., Jespersen, T. S., Marcus, C. M., \& Krogstrup, P. (2018). Engineering hybrid epitaxial InAsSb/Al nanowires for stronger topological protection. Physical Review Materials, 2(4), [044202 ]. https://doi.org/10.1103/PhysRevMaterials.2.044202

\section{General rights}

Copyright and moral rights for the publications made accessible in the public portal are retained by the authors and/or other copyright owners and it is a condition of accessing publications that users recognise and abide by the legal requirements associated with these rights.

- Users may download and print one copy of any publication from the public portal for the purpose of private study or research.

- You may not further distribute the material or use it for any profit-making activity or commercial gain

- You may freely distribute the URL identifying the publication in the public portal 


\title{
Engineering hybrid epitaxial InAsSb/Al nanowires for stronger topological protection
}

\author{
Joachim E. Sestoft, ${ }^{1}$ Thomas Kanne, ${ }^{1}$ Aske Nørskov Gejl, ${ }^{1}$ Merlin von Soosten, ${ }^{2}$ Jeremy S. Yodh, ${ }^{1}$ Daniel Sherman, ${ }^{1}$ \\ Brian Tarasinski, ${ }^{3}$ Michael Wimmer, ${ }^{3}$ Erik Johnson,,${ }^{2,4}$ Mingtang Deng, ${ }^{1}$ Jesper Nygård, ${ }^{2}$ Thomas Sand Jespersen, ${ }^{2}$ \\ Charles M. Marcus, ${ }^{1}$ and Peter Krogstrup ${ }^{1, *}$ \\ ${ }^{1}$ Center for Quantum Devices and Station Q Copenhagen, Niels Bohr Institute, University of Copenhagen, 2100 Copenhagen, Denmark \\ ${ }^{2}$ Center for Quantum Devices, Niels Bohr Institute, University of Copenhagen, 2100 Copenhagen, Denmark \\ ${ }^{3}$ QuTech and Kavli Institute of Nanoscience, Delft University of Technology, 2600 GA Delft, The Netherlands \\ ${ }^{4}$ Department of Wind Energy, Technical University of Denmark, Ris $\phi$ Campus, 4000 Roskilde, Denmark
}

(Received 29 December 2017; published 12 April 2018)

\begin{abstract}
The combination of strong spin-orbit coupling, large $g$ factors, and the coupling to a superconductor can be used to create a topologically protected state in a semiconductor nanowire. Here we report on growth and characterization of hybrid epitaxial InAsSb/Al nanowires, with varying composition and crystal structure. We find the strongest spin-orbit interaction at intermediate compositions in zinc-blende $\mathrm{InAs}_{1-x} \mathrm{Sb}_{x}$ nanowires, exceeding that of both InAs and InSb materials, confirming recent theoretical studies. We show that the epitaxial InAsSb/Al interface allows for a hard induced superconducting gap and $2 e$ transport in Coulomb charging experiments, similarly to experiments on InAs/Al and InSb/Al materials, and find measurements consistent with topological phase transitions at low magnetic fields due to large effective $g$ factors. Finally we present a method to grow pure wurtzite InAsSb nanowires which are predicted to exhibit even stronger spin-orbit coupling than the zinc-blende structure.
\end{abstract}

DOI: 10.1103/PhysRevMaterials.2.044202

Hybrid epitaxial semiconductor-superconductor interfaces hold potential for a variety of gateable superconducting experiments and applications. Semiconductor nanowires (NWs) with strong spin-orbit interaction (SOI) coupled to a superconductor are of particular interest [1-3] due to the prospect of hosting topologically protected Majorana zero modes (MZMs), which can be used for fault-tolerant quantum computing [4-7]. So far, signatures of MZMs which obey non-Abelian exchange statistics have been reported on binary III-V semiconductor/superconductor hybrids, e.g., InSb/Nb(TiN) and InAs/Al NWs [8-12], which possess the necessary properties for realizing a topological superconductor. Besides induced superconductivity, realizing topological protection and MZMs requires an applied magnetic field with a magnitude that depends on the effective SOI and $g$ factor of the hybrid system. Thus, realizing strong topological protection depends on the ability to engineer materials with the appropriate properties. A material platform which remains unexplored is ternary-based semiconductors such as InAsSb [13], which have been predicted to exhibit much stronger spin-orbit coupling than its binary compounds [14], and could potentially provide a material with sufficiently strong topological protection to realize topological quantum information applications. Moreover, InAsSb NWs are promising candidates for a variety of additional applications due to the tunable energy band gap (covering the whole IR spectrum, depending upon Sb concentration), making them highly promising in IR emission and detection along with ultrafast novel nano-optoelectronic devices [15-17].

\footnotetext{
*krogstrup@nbi.dk
}

Here we present structural, compositional, and electronic characterization of zinc-blende (ZB) and wurtzite (WZ) $\operatorname{InAs}_{1-x} \mathrm{Sb}_{x}$ NWs with and without epitaxially grown $\mathrm{Al}$. We characterize the crystal structure and composition with highresolution transmission electron microscopy (TEM) methods, and the electronic properties with low-temperature transport measurements. From measurements of weak antilocalization (WAL) on segmented $\mathrm{InAs}_{1-x} \mathrm{Sb}_{x}$ NWs of different Sb compositions $x$, we find a nonmonotonic dependence of spin-orbit length $\left(l_{\mathrm{SO}}\right)$ on the composition, with the smallest measured $l_{\text {SO }}$ at $x \sim 0.5$. For the hybrid InAsSb/Al NWs we find characteristic epitaxial semiconductor-superconductor structural ordering, where 3:2 lattice matched interfacial domains appear as the preferred interface for all compositions characterized. However, the interfaces do not appear as atomically sharp as in the case of the epitaxial InAs/Al interfaces [18], and residual dislocation arrays are present in the semiconductor as a result of strain relaxation relative to the strongly bound bicrystal interface. Despite these structural details, we do not observe any degradation of the induced gap from tunnel spectroscopy measurements on InAs $\mathrm{As}_{0.2} \mathrm{Sb}_{0.8} / \mathrm{Al} \mathrm{NWs}$. We study the density of states (DOS) as a function of applied magnetic field and show that Andreev bound states (ABS) merge to zero energy at relatively low magnetic fields due to large effective $g$ factors of the hybrid system. This is consistent with a topological phase transition as expected for materials with large SOI $[3,11,19]$. Coulomb charging experiments performed on NWs from the same growth batch show a transition from $2 e$ to $1 e$ charge periodicity at magnetic fields comparable to where the zero-energy states are observed in the DOS measurements. InAsSb NWs with ZB structure at intermediate concentrations of $\mathrm{Sb}$ were not measured as it was difficult to gate and pinch 

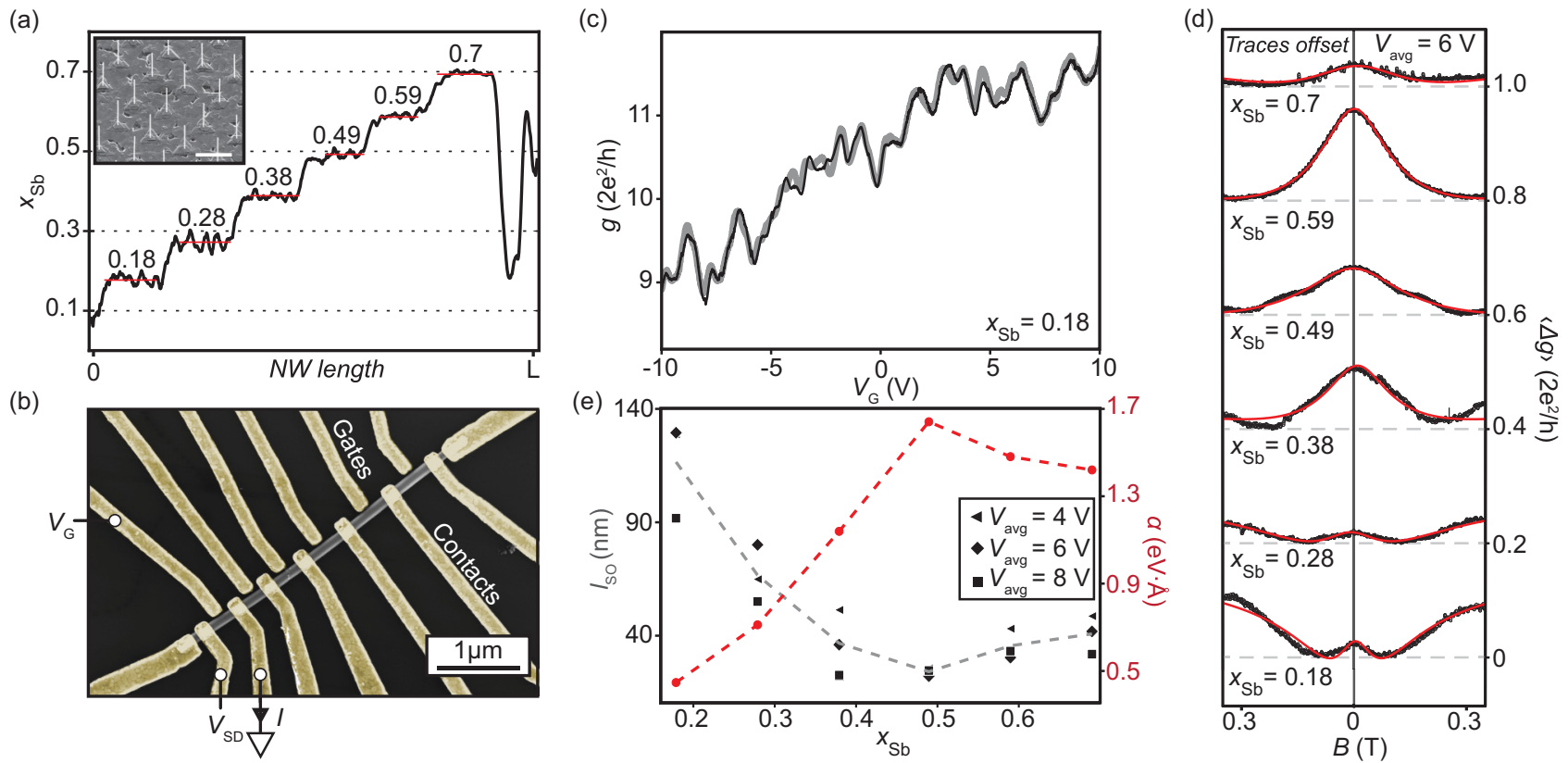

FIG. 1. (a) Sb concentration, $x$, as a function of NW length, $L$, with a steplike profile of the As/Sb molar fraction along the NW. The inset shows the NWs as grown on the substrate. (b) False-colored scanning electron micrograph of a typical device. Yellow, Ti/Au contacts and gates; gray, InAs ${ }_{1-x} \mathrm{Sb}_{x} \mathrm{NW} ; V_{\mathrm{SD}}$, the applied voltage bias; $I$, measured current; $V_{\mathrm{G}}$, gate voltage controlling the chemical potential on individual segments. (c) Trace/retrace (black/gray) of differential conductance for $x=0.18$ as a function of $V_{\mathrm{G}}$ showing aperiodic reproducible fluctuation with amplitude $\sim e^{2} / h$. (d) Averaged differential conductance change, $\langle\Delta g\rangle$, as a function of magnetic field showing suppression of the weak antilocalization effect around $B=0$ for all concentrations. Red overlay is the fit from Eq. (1), from which $l_{\text {So }}$ is extracted. (e) Spin-orbit length (left $y$ axis) as a function of Sb concentration. A noticeable minimum in $l_{\text {So }}$ is observed around $x \sim 0.5$, for the three different gate averaging amplitudes, $V_{\text {avg }}=4 \mathrm{~V}, 6 \mathrm{~V}$, and $8 \mathrm{~V}$. The Rashba coefficient (right $y$ axis) is a conversion of the average $l_{\text {So }}$ displaying a qualitative maximum around $x \sim 0.5$.

off with standard gating geometries. To circumvent this we present a method to grow WZ InAs $\mathrm{I}_{0.7} \mathrm{Sb}_{0.3} / \mathrm{Al} \mathrm{NWs}$, which is relevant both because of a lower electron affinity compared to $\mathrm{ZB}$ and because of a potentially higher SOI. We find an improved electrostatic gate response and measure a hard induced superconducting gap, comparable to what is measured in ZB NWs.

\section{SPIN-ORBIT INTERACTION IN InAs ${ }_{1-x} \mathrm{Sb}_{x} \mathrm{NWs}$}

To study the dependence of SOI strength on composition, we measure the magnetoconductance in axially segmented heterostructure NWs with six different compositions along the NW length. The NWs have a steplike compositional change along the growth direction as seen on the energydispersive x-ray spectroscopy (EDX) line scan in Fig. 1(a). The quantification of composition with TEM-EDX is done following the approach presented in the Supplemental Material of Ref. [20], and was confirmed with relative lattice spacing measures from high-resolution TEM (assuming Vegard's law). From measurements on multiple NWs, we find that each segment has a given length relative to the total NW length, which makes it possible to contact each individual segment by electron beam lithography (EBL), as seen in Fig. 1(b). Each contact is placed on the transition from one composition to the next, ensuring that every segment of the device has a given composition; see Sec. S3 of the Supplemental Material [21]. All measurements were carried out using standard ac lock-in measurements in a dilution refrigerator with a base temperature of $\sim 35 \mathrm{mK}$.

Two-terminal differential conductance measurements as a function of gate voltage, $V_{\mathrm{G}}$, were performed on each segment. An example of a trace/retrace is displayed for $x=0.18$ in Fig. 1(c). Reproducible conductance fluctuations are observed with an amplitude on the order of $e^{2} / h$ indicating universal conductance fluctuations (UCFs). This suggests that the phase coherence length, $l_{\phi}$, is on the order of or longer than the device length. We assume that the elastic scattering length, $l_{e}$, is shorter or comparable to $l_{\phi}$, which means that the transport resides in the diffusive regime [22-25]. A measure of the spin-orbit length is then extracted by fitting the magnetoconductance measurements, as similarly done on InAs and InSb NWs [23,26-30]. In this work the contribution from UCFs was averaged out by modulating the side gates with amplitudes, $V_{\text {avg }}$, using a sawtooth-shaped wave with a $2 \mathrm{~Hz}$ frequency. Here the averaging of the differential conductance was measured with a $372 \mathrm{~Hz}$ lock-in modulation over a $3 \mathrm{~s}$ period [31]. The typical scale of the UCFs was found from the correlation function to be $V_{\mathrm{c}} \sim 0.5 \mathrm{~V}$, and by using $V_{\text {avg }} \gg V_{\mathrm{c}}$ we ensure effective averaging over many UCF periods.

The average differential conductance, $\langle\Delta g\rangle$, is plotted as a function of magnetic field in Fig. 1(d) for all device segments using $V_{\text {avg }}=6 \mathrm{~V}$. A characteristic increase in $\langle\Delta g\rangle$ symmetrically around $B=0$ is observed indicating WAL which is canceled away from zero field $[32,33]$. In order to extract a measure of the phase coherence length, $l_{\phi}$, and the spin-orbit 
scattering length, $l_{\mathrm{SO}}$, we fit the measured magnetoconductance traces with a commonly used expression for the WAL correction to the conductance. We note that the extracted length scales are dependent on the model and fitting approach, rather than quantitative measures. However, we can reasonably compare relative length scales between the different compositions, and compare to literature values based on the same fitting approach. The model used here for the correction in the diffusive limit for small elastic scattering lengths is expressed as [34,35]

$$
\begin{aligned}
\Delta g(B)= & -\frac{2 e^{2}}{h L}\left[\frac{3}{2}\left(\frac{1}{l_{\phi}^{2}}+\frac{4}{3 l_{\mathrm{SO}}^{2}}+\frac{1}{D \tau_{\mathrm{B}}}\right)^{-1 / 2}\right. \\
& \left.-\frac{1}{2}\left(\frac{1}{l_{\phi}^{2}}+\frac{1}{D \tau_{\mathrm{B}}}\right)^{-1 / 2}\right] .
\end{aligned}
$$

Here, the magnetic dephasing time $\left(\tau_{\mathrm{B}}\right)$ is defined as $\tau_{\mathrm{B}}=\frac{C l_{\mathrm{m}}^{4}}{W^{2} D}$ where $D$ is the diffusion constant and the magnetic length, $l_{\mathrm{m}}=\left(\frac{\hbar}{e B}\right)^{1 / 2}$, is on the order of the NW diameter for the fitting range $0.35 \mathrm{~T}$, as in Refs. [23,27,28].

The prefactor, $C$, can in principle be computed numerically for a given geometry [29]. In general, it depends on details of the systems such as whether there is surface accumulation. However, modifications in the geometry typically only change $C$ by a factor of order 1, as we show in an example in Sec. S7 of the Supplemental Material [36]. Since we are mainly interested in the qualitative behavior of the SOI, we follow previously published work and use $C=3[23,37]$. Taking a fixed NW diameter $W=100 \mathrm{~nm}$ (as determined by SEM), we then use Eq. (1) to fit the magnetoconductance data measured with averaging voltages $V_{\text {avg }}=4,6$, and $8 \mathrm{~V}$. The red overlay in Fig. 1(d) shows the fit to the WAL data for all segments at $V_{\text {avg }}=6 \mathrm{~V}$. The mean standard deviation of goodness of fit of all data points shown in Fig. 1(e) is $\bar{\sigma} \sim 0.33 \mathrm{~nm}$, and we find $\chi^{2}=0.05$. We note that $l_{\phi}$ shows no apparent dependence on composition (see Sec. S4 of the Supplemental Material [38] for details). The extracted $l_{\text {SO }}$ is shown in Fig. 1(e) as a function of composition for all applied averaging voltages. Here, the smallest $l_{\mathrm{SO}}$ is obtained at $x \sim 0.5$, indicating a nonmonotonic dependence on composition and that a significantly smaller $l_{\mathrm{SO}}$ can be achieved compared to pure InAs and InSb NWs [23,26,28,29].

As these NWs are grown along the [111] direction with a pure ZB crystal structure, the Dresselhaus contribution from bulk inversion asymmetry is negligible [29] and therefore dominated by the Rashba contribution arising from structural inversion asymmetry. Therefore, $l_{\text {SO }}$ can be used as a measure of the Rashba spin-orbit strength. Following Refs. [27,39], we can rewrite the relation between the $l_{\mathrm{SO}}$ in a diffusive system into the spin-precession length $l_{\mathrm{R}}$ which is related to the Rashba coefficient as $l_{R}=\frac{\hbar^{2}}{2 m^{\star} \alpha_{R}}$. Using the estimate of the relation between $l_{\mathrm{SO}}=\frac{\sqrt{3} l_{R}^{2}}{W}$ we can relate the Rashba coefficient to the spin-orbit scattering lengths as $\alpha_{R}=\frac{\hbar^{2}}{2} m^{\star-1}\left(\frac{l_{S O} W}{\sqrt{C}}\right)^{-1 / 2}$. Estimates of $m^{\star}$ as a function of composition are found in Ref. [40]. In Fig. 1(e) we plot $\alpha$ as a function of composition and find the highest measured value of $1.65 \mathrm{eV} \AA$ at $x \sim 0.5$. This approach must be applied with caution since the obtained $l_{\mathrm{R}}$ is smaller than the diameter of the NW. However, for qualitative analysis of the Rashba contribution we expect this model to be applicable.

The strong SOI at intermediate compositions could have different origins. Ordering effects could lead to SOI enhancements as shown in recent $a b$ initio modeling performed on $\mathrm{InAs}_{1-x} \mathrm{Sb}_{x}$ NWs [14]. Here, a strongly enhanced SOI is found in the case of $\mathrm{CuPt}$ ordered stacking for compositions around $x=0.5$. We did not find any signature of such ordering in our TEM characterization, but small effects of such ordering could be reflected in the measurements, giving a minimum $l_{\mathrm{SO}}$ around $x=0.5$.

Previous studies have shown that the SOI measured by WAL can be influenced by the carrier density. Due to a weak gate coupling in these devices we cannot separate the carrier density and mobility of the samples. Thus the variation of $l_{\text {SO }}$ may be influenced by a dependence of carrier density on $x$. Nonetheless, we note that Rashba SOI exists even in the absence of external electric fields, arising from the intrinsic inversion asymmetry of the structure. Due to electrostatic gate tuning the electric fields in the InAsSb NWs gives rise to a Rashba SOI which is proportional to the semiconductor band gap [41], which is smallest for $x \sim 0.6$. Comparatively, in InSb NWs values of $\alpha \sim 1 \mathrm{eV} \AA$ were reported [29].

Since the band gap in $\mathrm{InAs}_{0.4} \mathrm{Sb}_{0.6}$ is roughly a factor 2 smaller than the band gap in InSb, we can expect around twice the value for $\alpha$ in InAsSb, which is in agreement with our measurements. Hence, we expect the enhanced SOI in $\mathrm{InAs}_{1-x} \mathrm{Sb}_{x} \mathrm{NW}$ s to be strongly dependent on the composition and the semiconductor band gap (especially around $x \sim 0.6$ ).

\section{EPITAXIAL RELATIONSHIP OF AI ON InAss ${ }_{1-x} \operatorname{Sb}_{x}$ NWs}

In the following section we focus on epitaxial growth of $\mathrm{Al}$ on InAsSb NWs at different compositions. As previously shown, a thin $\mathrm{Al}$ shell can be grown epitaxially on selected facets of InAs NWs at low temperatures [18,42,43], with a resulting hard induced superconducting gap [44], which makes them promising materials for gateable superconducting devices with low quasiparticle poisoning rates [45]. However, correlations between structural properties of the epitaxial match and the associated induced superconducting properties have so far not been studied in detail. By introducing Sb we change the lattice constant and crystal structure, which affects the relative orientation of the $\mathrm{Al}$ and bicrystal interfacial match. For NWs grown in the conventional [111]B/[0001]B growth direction, the crystal structure changes from $\mathrm{WZ}$ to $\mathrm{ZB}$, when exceeding a given small fraction of $\mathrm{Sb}$ [46]. Also, under the growth conditions used here, the facets change from InAs $\{1 \overline{1} 00\}_{\mathrm{WZ}}$ to InAsSb $\{1 \overline{1} 0\}_{\mathrm{ZB}}$, i.e., rotating $30^{\circ}$, and the lattice spacing increases linearly as a function of $\mathrm{Sb}$ concentration $[47,48]$.

In Figs. 2(a)-2(c) we show different bicrystal interfaces for different InAsSb compositions. The white and black arrows specify the crystal orientations for the InAsSb and Al, respectively. The schematics in the top-right corners indicate the viewing direction in relation to the NW morphology. Characteristic orientations of the $\mathrm{Al}$ grains are observed to be strongly dependent on the InAsSb composition. For low Sb concentrations, $x \sim 0.34$ [Fig. 2(a)], we find in-plane interfacial domains, which can be expressed in compact 
(a)

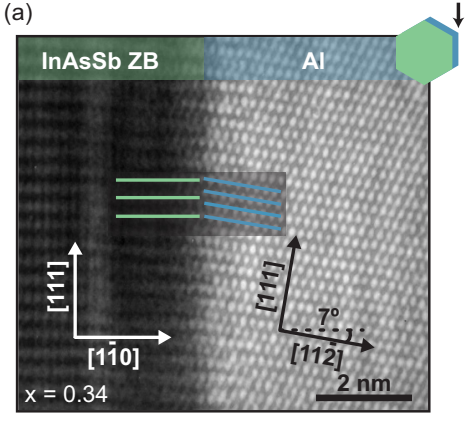

(b)

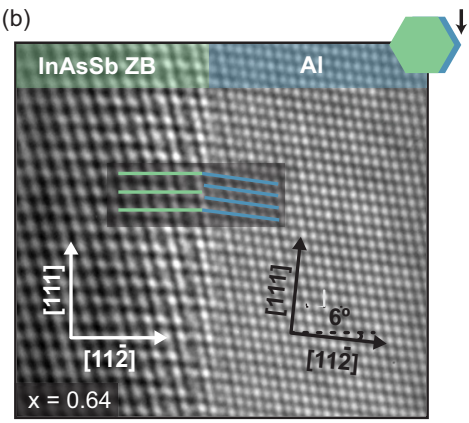

(c)

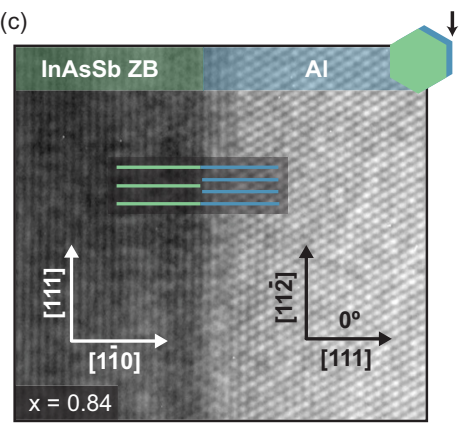

(e)

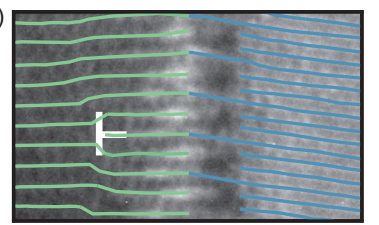

(d)
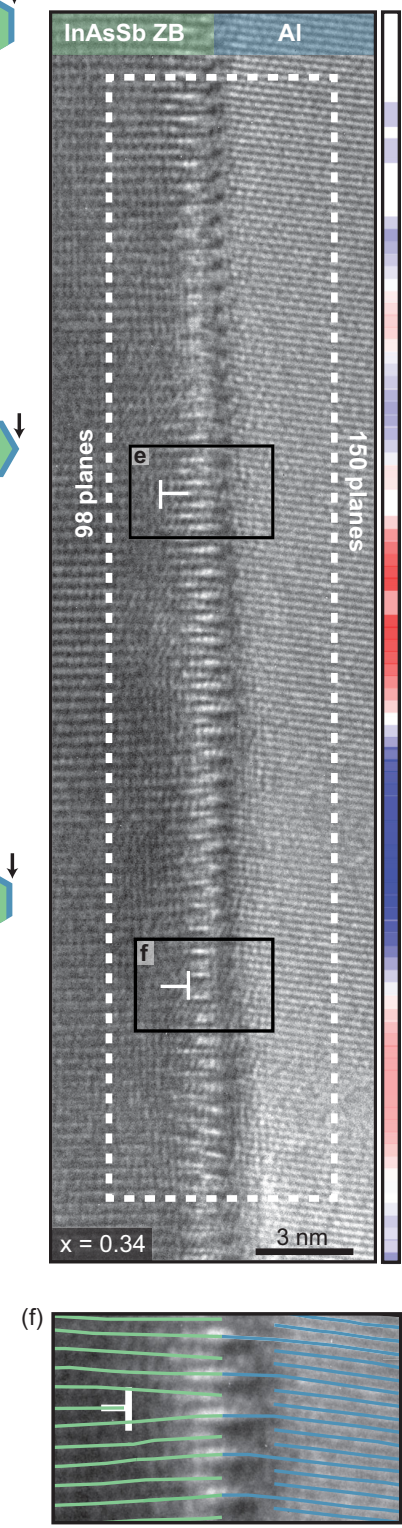

FIG. 2. (a) $7^{\circ}$ rotation between the $[1 \overline{1} 0]$ and the $[11 \overline{2}]$ directions in the semiconductor and the superconductor at $x=0.34$, respectively. Scale bar is the same for (a)-(c). (b) Rotation between the two [112] directions for $x=0.64$ is $6^{\circ}$. (c) No observed rotation between the [110] and [111] for $x=0.84$. (d) Bicrystal Burger circuit on a long segment of an interface for $x=0.34$ showing two edge dislocations with respect to $3: 2$ domain match. Color bar shows relative bending of the interplane distances with respect to a reference region away from the interface. Blue is upwards bending; red is downwards bending. (e), (f) The two dislocations from (d).

notation $\quad[18], \quad\left(\frac{3_{\mathrm{Al},[111]}}{2_{\mathrm{InAsSb},[111]}},-2.03 \%\right) \| \times\left(\frac{8_{\mathrm{Al},[110]}}{9_{\mathrm{InAsbb},[112]}}, 0.55 \%\right) \perp$, where the transverse domain match is estimated from atomic lattice modeling (see Sec. S4 of the Supplemental Material [38]). As the Sb concentration is increased [Fig. 2(b)] we find that the $\mathrm{Al}$ phase keeps an orientation that preserves an interfacial match with a $3: 2$ axial domain, $\left(\frac{3_{\mathrm{Al},[111]}}{2_{\mathrm{InAsSb},[11]}},-3.99 \%\right) \| \times$

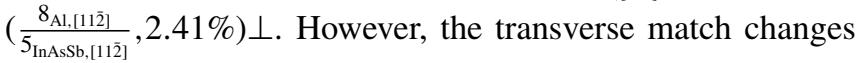

by aligning the $\mathrm{Al}[11 \overline{2}]$ planes along the semiconductor [112] planes. By increasing the Sb concentration even further [Fig. 2(c)] the Al phase attains the low interface energy domain of 3:2, with a low residual axial mismatch, $\left(\frac{3_{\mathrm{Al},[112]}}{2_{\mathrm{InASSb},[111]}}, 0.5 \%\right) \| \times$ $\left(\frac{8_{\mathrm{Al},[1 \overline{1}] 0]}}{9_{\mathrm{InASSb},[11 \overline{2}]}},-2.75 \%\right) \perp$.

The Al rotates discretely depending on the Sb concentration, with rotations occurring both radially and axially around the $(11 \overline{2})$ or (111) rotational axes. Examples of radial rotations are highlighted in Figs. 2(a) and 2(b), whereas Fig. 2(c) shows axial rotation but no radial rotation. Even small axial rotations obscure the visualization of the bicrystal match when imaging with TEM, because the InAsSb and Al will not simultaneously have a high symmetry zone axis. The Al orientation can be understood in terms of minimization of thermodynamic excess free energy under the constraints of kinetic barriers during grain growth [18]. The four main terms that contribute to the excess of the chemical potential are the surface energy, the grain boundaries, the semiconductor/superconductor interface, and related strain energies. Rotations increase the relative lattice plane distances at the interface and therefore may decrease the residual interfacial mismatch and lead to a higher ordered epitaxial interface and lower semiconductor/superconductor interfacial energy. If the interfacial bonding is strong, the semiconductor/superconductor interface term may dominate and lead to rotations in order to minimize the interfacial bonding energy. We propose that the discrete characteristics of the rotations are due to the fact that specific rotations give high symmetry cutting planes with low energy interfaces (see Sec. S5 of the Supplemental Material [49]). As shown above we generally find that the axial interfacial domain match seeks a 3:2 relation even though other lower ordered domains would give a lower residual mismatch. The 3:2 domain match is seen in the periodic interference effect (light/dark repeating pattern) along the interface. The dashed white rectangle in Fig. 2(d) shows an example of a bicrystal Burger circuit which counts 98 planes in the semiconductor and 150 in the superconductor away from the interface. This implies that the specific interface must have at least two residual misfit dislocations in order to acquire an interfacial 3:2 domain match. We find two types of dislocations and both occur in the InAsSb close to the interface. The edge dislocations are either associated with adding [Fig. 2(e)] or removing [Fig. 2(f)] a plane. Thus, the density of dislocations is here higher than predicted by the bicrystal Burger circuit. In the particular region of the interface shown in Fig. 2(d), we find four dislocations, three additions and one removal. The color plot on the right side of Fig. 2(d) shows the relative bending of the semiconductor planes in relation to a reference region away from the interface. Here, going from blue, which indicates downward bending planes, to red, which indicates upward bending planes, is associated with a dislocation (see Sec. S5 of the Supplemental Material [49]). The color plot shows the positions of the dislocations, which can be helpful because of bicrystal phase contrast smearing of the interface. The fact that all dislocations are observed inside the semiconductor, rather than at the interface, indicates a lower dislocation energy in the InAsSb bulk compared to the apparently strongly bonded 3:2 domain matched interface. This differs from epitaxial InAs/Al materials where no dislocations are found in the semiconductor and the Al phase appears fully relaxed with a $5 \mathrm{~nm}$ thick film 
[50]. Since the introduction of Sb is the only difference between the two types of NWs it is reasonable to assume that the Al-Sb bonds play a major role on this strong interfacial bonding. We note that even though we see dislocations appear to occur closer to the interface for Sb concentrations $x=0.34$ than for $x=0.64$, we have not been able to find a systematic trend for the dislocation depth as a function of composition.

\section{TUNNEL-PROBING InAs $s_{0.2} \mathrm{Sb}_{0.8} / \mathrm{Al}$ NWs}

In the following section we investigate the superconducting transport properties of the hybrid $\operatorname{InAs}_{0.2} \mathrm{Sb}_{0.8} / \mathrm{Al}$ material using two device geometries: a normal-metal insulator-superconductor (NIS) device and a normal-metal insulator-superconductor insulator-normal metal (NISIN) device [Figs. 3(a) and 4(a), respectively]. The so-called insulator part is a segment of InAsSb where the Al is selectively etched away to enable carrier depletion by electrostatic gating. We note that we were not able to deplete carriers in devices with $x$ between $0.3-0.7$, using standard gating geometries, and hence focus on devices with $x=0.8$.

Data from tunneling conductance spectra of the bottomgated NIS device are shown in Fig. 3(b). By adjusting the voltage applied on the junction, $V_{\text {cut }}$, we restrict the device

(a)
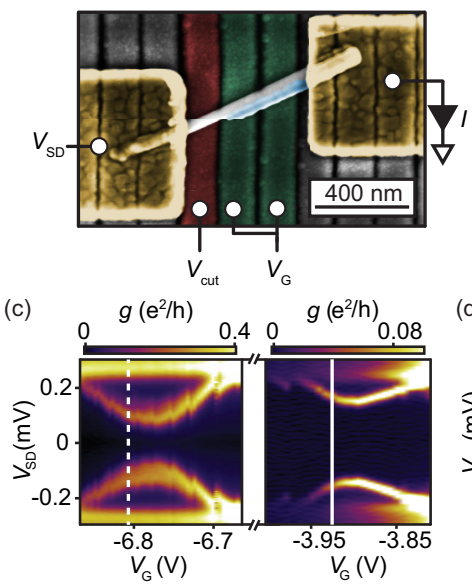

(e)
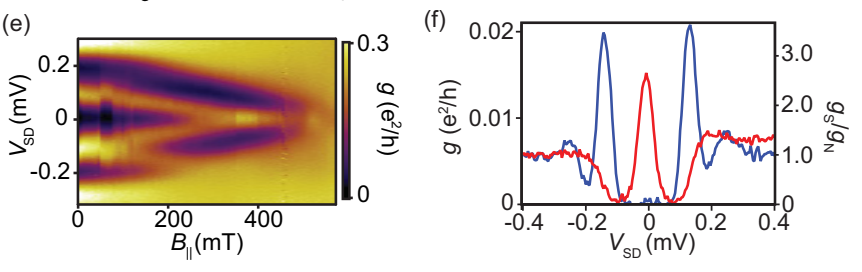

FIG. 3. (a) False-colored scanning electron micrograph of a NIS device. (b) Differential conductance as a function of $V_{\mathrm{SD}}$ plotted on a linear (blue) and logarithmic scale (red) showing a highly suppressed differential conductance within $\Delta= \pm 230 \mu \mathrm{eV}$. (c) Conductance as a function of $V_{\mathrm{SD}}$ and $V_{\mathrm{G}}$ showing two symmetric ABS within $\Delta \sim 230$ $\mu \mathrm{eV}$ at two different gate configurations. Unbroken and dashed lines indicate the gate configuration for (d) and (e), respectively. (d), (e) Conductance as a function of $V_{\mathrm{SD}}$ and $B_{\|}$showing the magnetic field evolution of two ABS that merge and form zero-bias peaks at $\sim 350$ and $400 \mathrm{mT}$, respectively. (f) Conductance as a function of $V_{\mathrm{SD}}$ corresponding to line cuts (blue and red squares) seen in (d); shows the zero-bias peak with strongly suppressed differential conductance symmetrically around the peak, signature of a hard topological gap. in the tunneling regime with a conductance well below the conductance quantum $\left(g \ll 2 e^{2} / h\right)$. From the tunneling spectrum, as a function of source-drain voltage, we find the superconducting gap, $\Delta$, to be approximately $\sim 230 \mu \mathrm{eV}$. The differential conductance in the gap is suppressed by a factor of approximately $10^{2}$ compared to the conductance outside of the gap, which is similar to the gap hardness reported on epitaxial InAs/Al NWs [44], and seems to be attributed to the clean superconductor-semiconductor interface rather than specific epitaxial lattice matching. We note that while the critical field is comparable to what we typically find for similar Al thicknesses on InAs/Al NWs, the induced superconducting gap of $\Delta \sim 230$ $\mu \mathrm{eV}$ is significantly larger.

In Fig. 3(c) differential conductance as a function of gate voltage, $V_{\mathrm{G}}$, and $V_{\mathrm{SD}}$ shows two pairs of $\mathrm{ABS}$ at two very different gate configurations extending out of the continuum symmetrically around $V_{\mathrm{SD}}=0$. For most gate configurations at zero field the superconducting gap appears hard and empty of states. By tuning $V_{\text {cut }}$ the coupling, $\Gamma$, between the lead and the proximitized segment can be controlled, as also evident by the difference in the magnitude of differential conductance between the left and right plot.

For gate voltages corresponding to the dashed and unbroken line cuts in Fig. 3(c) left and right panels, Figs. 3(e) and 3(d) show the respective DOS evolution in a parallel magnetic field along the NW axis. The parallel magnetic field orientation is found as similarly described in the methods section of Ref. [12]. The ABS merge and pin to $V_{\mathrm{SD}}=0$ at magnetic fields around $B \sim 350$ and $400 \mathrm{mT}$, respectively. This occurs at relatively low fields $[8,9,11]$ due to large effective $g$ factors of the hybrid system, in this case extracted to be on the order of 10. Like the overall conductance, also the bound state conductance and zero-bias peak (ZBP) height depend strongly on $V_{\text {cut }}$. For the ZBPs it was not possible to exceed $0.3 \times 2 e^{2} / h$ by opening $V_{\text {cut }}$ before the gap softened and the overall conductance increased. This indicates that the cutter region where the $\mathrm{Al}$ is etched away does not fulfill resonant tunneling. Ideal tunneling was recently observed in two-dimensional hybrid materials with a thin etch stop layer between the $\mathrm{Al}$ and the InAs, and also in hybrid NWs with "shadowed" junctions [51,52]. The ZBPs prevail until the closing of the superconducting gap at critical fields of the $\mathrm{Al}$ at $B_{\mathrm{c}} \sim 600-800 \mathrm{mT}$. The observed critical fields are low compared to similar experiments conducted on InAs NWs proximitized by $\mathrm{Al}$ which yielded critical fields of $B_{\mathrm{c}} \sim 2 \mathrm{~T}$ with a film thickness of $7 \mathrm{~nm}$ [11]. We attribute the lower critical fields to the average Al film thickness which in our case is about $15 \mathrm{~nm}$ [53]. The blue and red squares in Fig. 3(d) correspond to the two line traces in Fig. 3(f), where the normalized differential conductance as a function of $V_{\mathrm{SD}}$ at $B_{\|}=0$ and $430 \mathrm{mT}$ is plotted. We find that the gap stays hard around the ZBP at $B_{\|}=430 \mathrm{mT}$, with a gap of the order of $\Delta$. Extracted $g$ factors of the hybrid system at various gate configurations are generally extracted to be between 10 and 20 as shown in Sec. S8 of the Supplemental Material [54].

\section{COULOMB SPECTROSCOPY ON InAs ${ }_{0.2} \mathrm{Sb}_{0.8} / \mathrm{Al} \mathrm{NWs}$}

Additional transport measurements were performed on the same batch of $\mathrm{InAs}_{0.2} \mathrm{Sb}_{0.8} / \mathrm{Al} \mathrm{NWs}$ using a normal-metal 


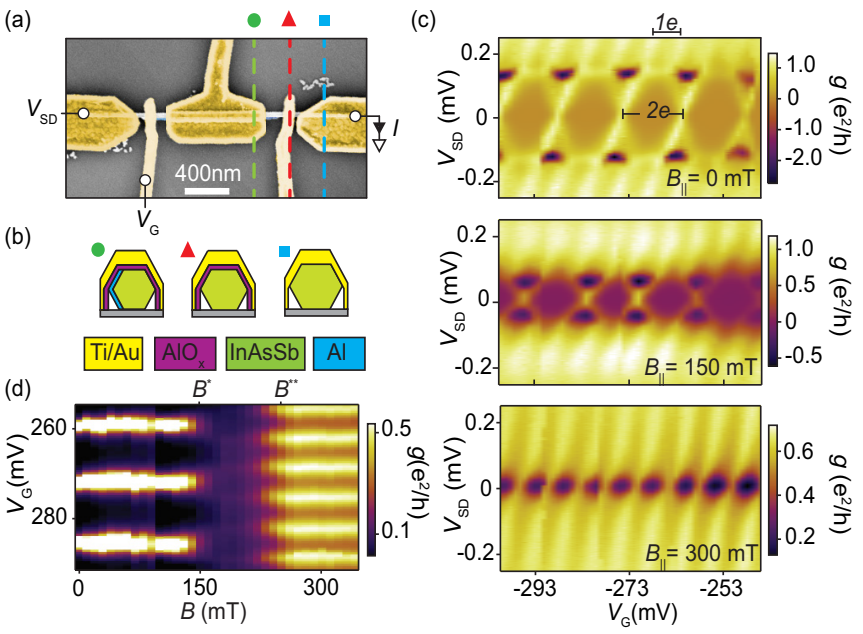

FIG. 4. (a) False-colored SEM image of the measured NISIN device. (b) Cartoon of the cross sections indicated in (a) by three colored dashed lines illustrates the top-gate material sequencing. (c) Differential conductance, $g$, as a function of $V_{\mathrm{G}}$ and $V_{\mathrm{SD}}$ showing $2 e$-spaced Coulomb diamonds within $\Delta \sim 0.15 \mathrm{meV}$. For $V_{\mathrm{SD}}>0.15 \mathrm{mV}, 1 e$ periodic Coulomb resonances are evident, verifying Cooper pair tunneling within $V_{\mathrm{SD}} \sim \pm 0.15 \mathrm{mV}$. (d) Differential conductance at zero bias as a function of $V_{\mathrm{G}}$ and $B_{\|}$. Splitting from $2 e$ to $1 e$ initiates at $B^{*}=B_{\|}=150 \mathrm{mT}$ and is evenly $1 e$ spaced at $B^{* *}=B_{\|}=250 \mathrm{mT}$, suggesting a smooth transition from $2 e$ to $1 e$.

insulator-superconductor insulator-normal metal (NISIN) geometry. In Fig. 4(a) we show a scanning electron micrograph of a measured NISIN device with a superconductor island length of $L=800 \mathrm{~nm}$. In this device the gates are realized as top gates using $\mathrm{AlO}_{x}$ as a dielectric. Schematics of the cross sections of the different material stacking sequences of the device are seen in Fig. 4(b).

Differential conductance as a function of $V_{\mathrm{SD}}$ and $V_{\mathrm{G}}$ at different magnitudes of parallel magnetic fields are shown in Fig. 4(c). Evenly spaced Coulomb diamonds are observed as well as differential conductance resonances above the superconducting gap. At $B_{\|}=0$ and $V_{\mathrm{SD}}=0$ each Coulomb resonance occurs at double the gate voltage separation compared to the period observed above the superconducting gap. The period doubling within the superconducting gap implies Cooper pair tunneling of charge $2 e$ as opposed to the $1 e$ periodicity above the superconducting gap. As previously reported from experiments on superconductor islands [45,55,56], we see regions with strong negative differential conductance (NDC) at bias voltages where the $2 e$ to $1 e$ transitions occur. At $B=150$ $\mathrm{mT}$ the $2 e$ periodic Coulomb diamonds are still observed, however with a decreased superconducting gap and magnitude of the NDC. At $B=300 \mathrm{mT}$, well below the critical field, $B_{\mathrm{c}}$, of the $\mathrm{Al}$, the Coulomb resonances exhibit a $1 e$ periodicity. This is consistent with the energy of the odd charge state being lowered sufficiently by the Zeeman energy in order for the $1 e$ periodicity to become dominant [12]. We speculate that the lack of visible oscillations in the even-odd energy difference, as observed in Ref. [12], could be due to the combination of a relatively large island size and high SOI for this material, producing a minimal overlap between the Majorana wave functions. Tunneling from the bound state to the Bardeen-Cooper-Schrieffer continuum in the $\mathrm{Al}$ gives rise to NDC due to transport blockade by electron/hole excitations. When the quasiparticle relaxes from the continuum back into the bound state and escapes to the leads the transport blockade is lifted. This way large NDC indicates a relatively long quasiparticle relaxation time as explained in Ref. [45].

Figure 4(d) shows the evolution of the zero-bias Coulomb peaks in a parallel magnetic field. A distinct $2 e$ peak spacing is evident until $B=150 \mathrm{mT}$ where the splitting initiates. Noticeably, the intensity of the zero-bias resonances decreases drastically as it evolves into a $1 e$ periodicity between $B^{*}$ and $B^{* *}$. From $B=250 \mathrm{mT}$ the evenly spaced Coulomb resonances regain intensity but with a $1 e$ evenly spaced periodicity and no signatures of even-odd intensity variance-again consistent with transport through topologically protected MZMs [57,58]. The splitting is completed well below the closing of the induced superconducting gap, which is spectroscopically extracted to be between $B_{\mathrm{c}} \sim 600-800 \mathrm{mT}$ (depending on gate configuration). For example, residual superconductivity is still clearly visible at $B=300 \mathrm{mT}$, as seen in Fig. 4(c).

\section{WZ InAss ${ }_{0.3} \mathrm{Sb}_{0.7} / \mathrm{Al} \mathrm{NWs}$}

As discussed above, we find that $\mathrm{ZB}$ InAs ${ }_{1-x} \mathrm{Sb}_{x} \mathrm{NWs}$ exhibit a weak electrostatic gate response for a range of compositions. The electron affinity of the InAsSb material crucially influences the filling of the conduction band, and therefore how easily the NW can be gated and possibly depleted. As the electron affinity of III-V materials typically is higher in ZB than WZ [59], we expect that InAsSb NWs with a WZ crystal structure could improve gateability. Since it is challenging to form WZ phases in NWs containing Sb, because of a large bulk cohesive energy difference between ZB and WZ $[60,61]$, we grow from a WZ basis by initiating growth from the (1100) side facets of [0001] InAs WZ NWs, as explained in Refs. [20,62]. This is illustrated in the inset in Fig. 5(a). As long as the NWs grow layer by layer in the [1 100$]$ direction, the crystal structure is locked in the parent WZ crystal basis, and can in this case not change to $\mathrm{ZB}$ without introducing a high energy incoherent interface. After initiating the [1100] InAs growth, we gradually introduce $\mathrm{Sb}$ over a segment of $\sim 1 \mu \mathrm{m}$ to avoid secondary kinking, and continue the InAsSb growth for $\sim 5 \mu \mathrm{m}$. We terminate the growth with an epitaxially grown shell of Al on the top facet. The NWs as-grown are seen in Fig. 5(a). A high-resolution TEM micrograph of the bicrystal interfacial match between the WZ $\mathrm{InAs}_{0.7} \mathrm{Sb}_{0.3} \mathrm{NW}$ and the $\mathrm{Al}$ shell is seen in Fig. 5(b). Similarly to the ZB hybrids presented in Fig. 2, we find a well-defined and epitaxial interface with a 3:2 domain match along the growth direction. In contrast to the similar InAs/Al NWs [18], the residual mismatch along the [1100] direction is negative and thus bending the NW upwards as evident in Fig. 5(c). In Fig. 5(c) we show a false-colored SEM micrograph of the InAs $\mathrm{An}_{0.7} \mathrm{Sb}_{0.3} \mathrm{WZ} \mathrm{NW}$ connected by normal-metal leads. The device geometry is similar to the geometry shown in Fig. 3, except side gates are used instead of bottom gates. The inset shows an EDX map with normalized intensities for In, As, and Sb, performed on the last part of a kinked NW as illustrated by the white square. This verifies a constant composition along the growth direction with no detectable $\mathrm{Sb}$ shell formation and a quantified composition 

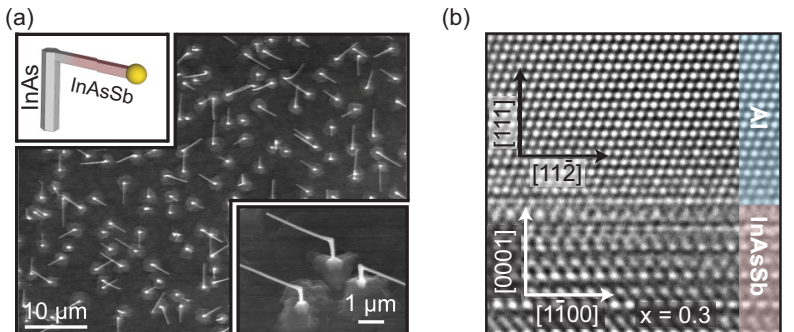

(c) $V_{\mathrm{SD}}$

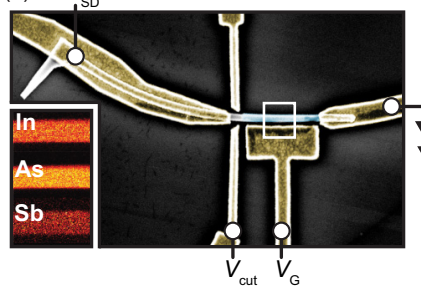

(d)

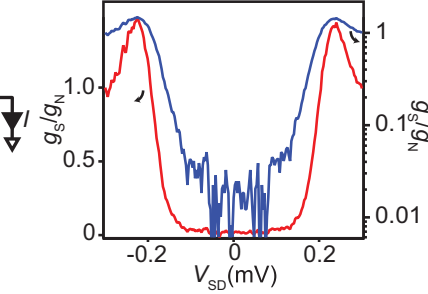

FIG. 5. (a) Top-view SEM micrograph of the NWs grown in the six directions parallel to the substrate. Inset show a $45^{\circ}$ tilted SEM micrograph of three kinked structures. (b) HR-TEM micrograph of the InAsSb/Al interface shows a coherent epitaxial crystal match. The black and white arrows indicate the orientations of the hybrid system, where a 3:2 domain match with no rotation is observed. (c) False-colored SEM micrograph of a NIS device composed of normal-metal leads connected to an $\mathrm{InAs}_{0.7} \mathrm{Sb}_{0.3}$ WZ NW grown along the [1100] direction. Inset shows normalized EDX intensities for In, As, and $\mathrm{Sb}$ from a region of the NW shown by the white box. (d) Differential conductance as a function of $V_{\mathrm{SD}}$ plotted on a linear (red trace) and logarithmic scale (blue trace) showing a superconducting hard gap with highly suppressed differential conductance within $\Delta= \pm 225 \mu \mathrm{eV}$.

of $x=0.3$. In Fig. 5(d) the left $y$ axis shows normalized differential conductance as a function of $V_{\mathrm{SD}}$ displaying a hardgap profile in the DOS. The right $y$ axis shows the same normalized differential conductance plotted on a logarithmic scale where the differential conductance within $\Delta=0.225 \mathrm{meV}$ is suppressed by a factor of $\sim 80$. These findings are comparable to transport measurements performed on the $\mathrm{ZB} \mathrm{InAs} \mathrm{Is}_{0.2} \mathrm{Sb}_{0.8}$ NWs. Assuming that the WZ electron affinity as a function of composition follows that of $\mathrm{ZB}$, a composition of $x=0.3$ would have an effective maximum [63]; thus it is likely that all compositions of $\mathrm{WZ} \operatorname{InAs}_{1-x} \mathrm{Sb}_{x} \mathrm{NWs}$ would be depletable simply by applying standard side gates. Additional advantages to WZ NWs grown in the [1 1100$]$ direction could be a contribution to the SOI from the Dresselhaus spin-orbit term that is linear in momentum, arising solely due to asymmetry in the crystal structure [31].

In conclusion, we show from WAL measurements that the SOI as a function of composition in InAs $\mathrm{Is}_{1-x} \mathrm{Sb}_{x}$ NWs exhibits a nonmonotonic behavior with a maximum in the Rashba spin-orbit coefficient at $x \sim 0.5$. By growing $\mathrm{Al}$ shells in situ on InAs ${ }_{1-x} \mathrm{Sb}_{x}$ NWs with varied compositions we find an epitaxial relationship with a specific low energy interfacial domain of 3:2 in the axial direction for all compositions. Axial and radial $\mathrm{Al}$ rotations combined with interfacial edge dislocations seem to reduce the contribution to the excess energy. Coulomb charging and tunneling measurements on $\operatorname{InAs}_{0.2} \mathrm{Sb}_{0.8} / \mathrm{Al}$ hybrid NWs show hard gap induced superconductivity, as well as data consistent with topological superconductivity.

From the tunneling experiments we find a ZBP protected by a hard superconducting gap. Additionally, from the Coulomb charging experiments we find strong NDC indicating long quasiparticle poisoning times.

In the end we present a method to grow InAsSb NWs with WZ structure, which shows an improved electrostatic response for $\mathrm{InAs}_{0.7} \mathrm{Sb}_{0.3} \mathrm{NWs}$ grown along the [1100] direction. The strong Rashba SOI, the good electrostatic response, the welldefined epitaxial superconductor-semiconductor match, and the potential addition to an even higher SOI due to a potential Dresselhaus contribution are all ingredients that make WZ $\operatorname{InAs}_{0.5} \mathrm{Sb}_{0.5} / \mathrm{Al}$ hybrid NWs a promising material for further studies on topological superconductivity.

\section{ACKNOWLEDGMENTS}

This project was funded by Microsoft Station Q, the Danish National Science Research Foundation, the Carlsberg Foundation, the Villum Foundation, and the European Research Council (ERC), which supported the research under Grant Agreement No. 716655 (HEMs-DAM). We thank Esben Bork Hansen for fruitful discussions, and Claus B. Sørensen and Shivendra Upadhyay for technical assistance.

J.E.S., T.K., and A.N.G. contributed equally to this work.
[1] Y. Oreg, G. Refael, and F. von Oppen, Phys. Rev. Lett. 105, 177002 (2010).

[2] R. M. Lutchyn, J. D. Sau, and S. Das Sarma, Phys. Rev. Lett. 105, 077001 (2010).

[3] S. Das Sarma, M. Freedman, and C. Nayak, npj Quantum Information 1, 15001 (2015).

[4] M. Freedman, A. Kitaev, M. Larsen, and Z. Wang, Bull. Am. Math. Soc. 40, 31 (2003).

[5] N. Read and D. Green, Phys. Rev. B 61, 10267 (2000).

[6] A. Y. Kitaev, Phys. Usp. 44, 131 (2001).

[7] L. Fu and C. L. Kane, Phys. Rev. Lett. 100, 096407 (2008).

[8] V. Mourik, K. Zuo, S. M. Frolov, S. Plissard, E. Bakkers, and L. P. Kouwenhoven, Science 336, 1003 (2012).
[9] M. Deng, C. Yu, G. Huang, M. Larsson, P. Caroff, and H. Xu, Nano Lett. 12, 6414 (2012).

[10] A. Das, Y. Ronen, Y. Most, Y. Oreg, M. Heiblum, and H. Shtrikman, Nat. Phys. 8, 887 (2012).

[11] M. Deng, S. Vaitiekènas, E. B. Hansen, J. Danon, M. Leijnse, K. Flensberg, J. Nygård, P. Krogstrup, and C. M. Marcus, Science 354, 1557 (2016).

[12] S. M. Albrecht, A. Higginbotham, M. Madsen, F. Kuemmeth, T. S. Jespersen, J. Nygård, P. Krogstrup, and C. Marcus, Nature (London) 531, 206 (2016).

[13] L. Namazi, S. G. Ghalamestani, S. Lehmann, R. R. Zamani, and K. A. Dick, Nanotechnology 28, 165601 (2017).

[14] G. W. Winkler, Q. Wu, M. Troyer, P. Krogstrup, and A. A. Soluyanov, Phys. Rev. Lett. 117, 076403 (2016). 
[15] D. Cheung, A. Andrews, E. Gertner, G. Williams, J. Clarke, J. Pasko, and J. Longo, Appl. Phys. Lett. 30, 587 (1977).

[16] J. Kim, S. Kim, D. Wu, J. Wojkowski, J. Xu, J. Piotrowski, E. Bigan, and M. Razeghi, Appl. Phys. Lett. 67, 2645 (1995).

[17] J. Svensson, N. Anttu, N. Vainorius, B. M. Borg, and L.-E. Wernersson, Nano Lett. 13, 1380 (2013).

[18] P. Krogstrup, N. Ziino, W. Chang, S. Albrecht, M. Madsen, E. Johnson, J. Nygård, C. Marcus, and T. Jespersen, Nat. Mater. 14, 400 (2015).

[19] R. Lutchyn, E. Bakkers, L. Kouwenhoven, P. Krogstrup, C. Marcus, and Y. Oreg, arXiv:1707.04899.

[20] P. Krogstrup, J. Yamasaki, C. B. Sørensen, E. Johnson, J. B. Wagner, R. Pennington, M. Aagesen, N. Tanaka, and J. Nygård, Nano Lett. 9, 3689 (2009).

[21] See Supplemental Material at http://link.aps.org/supplemental/ 10.1103/PhysRevMaterials.2.044202 for details on device fabrication.

[22] B. L. Al'tshuler, JETP Lett. 41, 648 (1985).

[23] A. E. Hansen, M. T. Björk, C. Fasth, C. Thelander, and L. Samuelson, Phys. Rev. B 71, 205328 (2005).

[24] P. A. Lee and A. D. Stone, Phys. Rev. Lett. 55, 1622 (1985).

[25] P. A. Lee, A. D. Stone, and H. Fukuyama, Phys. Rev. B 35, 1039 (1987).

[26] S. Estévez Hernández, M. Akabori, K. Sladek, C. Volk, S. Alagha, H. Hardtdegen, M. G. Pala, N. Demarina, D. Grützmacher, and T. Schäpers, Phys. Rev. B 82, 235303 (2010).

[27] P. Roulleau, T. Choi, S. Riedi, T. Heinzel, I. Shorubalko, T. Ihn, and K. Ensslin, Phys. Rev. B 81, 155449 (2010).

[28] S. Dhara, H. S. Solanki, V. Singh, A. Narayanan, P. Chaudhari, M. Gokhale, A. Bhattacharya, and M. M. Deshmukh, Phys. Rev. B 79, 121311 (2009).

[29] I. van Weperen, B. Tarasinski, D. Eeltink, V. S. Pribiag, S. R. Plissard, E. P. A. M. Bakkers, L. P. Kouwenhoven, and M. Wimmer, Phys. Rev. B 91, 201413(R) (2015).

[30] L. Wang, J. Guo, N. Kang, D. Pan, S. Li, D. Fan, J. Zhao, and H. Xu, Appl. Phys. Lett. 106, 173105 (2015).

[31] T. S. Jespersen, P. Krogstrup, A. M. Lunde, R. Tanta, T. Kanne, E. Johnson, and J. Nygård, Phys. Rev. B 97, 041303(R) (2018).

[32] S. Hikami, A. I. Larkin, and Y. Nagaoka, Prog. Theor. Phys. 63, 707 (1980)

[33] G. Bergmann, Phys. Rev. Lett. 48, 1046 (1982).

[34] C. Kurdak, A. M. Chang, A. Chin, and T. Y. Chang, Phys. Rev. B 46, 6846 (1992).

[35] B. L. Al'tshuler and A. G. Aronov, Pis'ma Zh. Eksp. Teor. Fiz. 33, 515 (1981) [JETP Lett. 33, 499 (1981)].

[36] See Supplemental Material at http://link.aps.org/supplemental/ 10.1103/PhysRevMaterials.2.044202 for details on simulations of the prefactor, $C$.

[37] C. Beenakker and H. van Houten, Solid State Phys. 44, 1 (1991).

[38] See Supplemental Material at http://link.aps.org/supplemental/ 10.1103/PhysRevMaterials.2.044202 for details on extracted phase coherence lengths.

[39] T. Schäpers, V. A. Guzenko, M. G. Pala, U. Zülicke, M. Governale, J. Knobbe, and H. Hardtdegen, Phys. Rev. B 74, 081301(R) (2006).

[40] I. Vurgaftman, J. Meyer, and L. Ram-Mohan, J. Appl. Phys. 89, 5815 (2001).
[41] R. Winkler, Spin-Orbit Coupling Effects in Two-Dimensional Electron and Hole Systems (Springer, Berlin, 2003), p. 61.

[42] N. A. Güsken, T. Rieger, B. Bennemann, E. Neumann, M. I Lepsa, T. Schäpers, and D. Grützmacher, Nanoscale 9, 16735 (2017).

[43] S. Gazibegovic, D. Car, H. Zhang, S. C. Balk, J. A. Logan, M. W. de Moor, M. C. Cassidy, R. Schmits, D. Xu, G. Wang et al., Nature (London) 548, 434 (2017).

[44] W. Chang, S. Albrecht, T. Jespersen, F. Kuemmeth, P. Krogstrup, J. Nygård, and C. Marcus, Nat. Nanotechnol. 10, 232 (2015).

[45] A. P. Higginbotham, S. M. Albrecht, G. Kiršanskas, W. Chang, F. Kuemmeth, P. Krogstrup, T. S. Jespersen, J. Nygård, K. Flensberg, and C. M. Marcus, Nat. Phys. 11, 1017 (2015).

[46] H. Potts, M. Friedl, F. Amaduzzi, K. Tang, G. Tutuncuoglu, F. Matteini, E. Alarcon Llado, P. C. McIntyre, and A. Fontcuberta i Morral, Nano Lett. 16, 637 (2015).

[47] L. Lugani, D. Ercolani, L. Sorba, N. Sibirev, M. Timofeeva, and V. Dubrovskii, Nanotechnology 23, 095602 (2012).

[48] D. Ercolani, M. Gemmi, L. Nasi, F. Rossi, M. Pea, A. Li, G. Salviati, F. Beltram, and L. Sorba, Nanotechnology 23, 115606 (2012).

[49] See Supplemental Material at http://link.aps.org/supplemental/ 10.1103/PhysRevMaterials.2.044202 for details on bicrystal relations.

[50] J. Shabani, M. Kjaergaard, H. Suominen, Y. Kim, F. Nichele, K. Pakrouski, T. Stankevic, R. M. Lutchyn, P. Krogstrup, R. Feidenhans'l et al., Phys. Rev. B 93, 155402 (2016).

[51] F. Nichele, A. C. Drachmann, A. M. Whiticar, E. C. O'Farrell, H. J. Suominen, A. Fornieri, T. Wang, G. C. Gardner, C. Thomas, A. T. Hatke et al., Phys. Rev. Lett. 119, 136803 (2017).

[52] H. Zhang, C.-X. Liu, S. Gazibegovic, D. Xu, J. A. Logan, G. Wang, N. van Loo, J. D. Bommer, M. W. de Moor, D. Car et al., Nature (2018), doi:10.1038/nature26142.

[53] R. Meservey and P. Tedrow, J. Appl. Phys. 42, 51 (1971).

[54] See Supplemental Material at http://link.aps.org/supplemental/ 10.1103/PhysRevMaterials.2.044202 for extraction of effective $g$ factors.

[55] F. W. J. Hekking, L. I. Glazman, K. A. Matveev, and R. I. Shekhter, Phys. Rev. Lett. 70, 4138 (1993).

[56] J. M. Hergenrother, M. T. Tuominen, and M. Tinkham, Phys. Rev. Lett. 72, 1742 (1994).

[57] L. Fu, Phys. Rev. Lett. 104, 056402 (2010).

[58] R. Hützen, A. Zazunov, B. Braunecker, A. L. Yeyati, and R. Egger, Phys. Rev. Lett. 109, 166403 (2012).

[59] C.-Y. Yeh, Z. W. Lu, S. Froyen, and A. Zunger, Phys. Rev. B 46 , 10086 (1992).

[60] V. Dubrovskii, Nucleation Theory and Growth of Nanostructures (Springer, Berlin, Heidelberg, 2014), pp. 275-395.

[61] S. G. Ghalamestani, S. Lehmann, and K. A. Dick, Nanoscale 8, 2778 (2016).

[62] F. Krizek, T. Kanne, D. Razmadze, E. Johnson, J. Nygård, C. M. Marcus, and P. Krogstrup, Nano Lett. 17, 6090 (2017).

[63] P. Webster, N. Riordan, S. Liu, E. Steenbergen, R. Synowicki, Y.-H. Zhang, and S. Johnson, J. Appl. Phys. 118, 245706 (2015). 